\title{
Body composition and serum lipid profile of Royal Navy submariners before and after a three month deployment
}

\author{
F. Gunner ${ }^{1}$, M. Lindsay ${ }^{1}$, P.E.H. Brown ${ }^{1}$, A.M. Shaw ${ }^{1}$, S.E. Britland ${ }^{1}$, T. Davey ${ }^{1}$, \\ S.A. Lanham-New ${ }^{2}$, B.A. Griffin ${ }^{2}$ and J.L. Fallowfield ${ }^{1}$ \\ ${ }^{1}$ The Institute of Naval Medicine, Gosport, Hampshire and \\ ${ }^{2}$ Department of Nutritional Sciences, School of Biosciences and Medicine, Faculty of Health and Medical Sciences, \\ University of Surrey, Guildford, Surrey
}

Risk factors for the development of metabolic syndrome include physical inactivity, obesity and a diet high in fat and carbohydrate ${ }^{(1)}$. Royal Navy (RN) submarine deployments involve prolonged periods of submersion with limited opportunity for physical exercise and compromised supply of fresh food. To test the hypothesis that these environmental conditions may contribute to poor cardiometabolic health, the impact of a submarine deployment on the body composition and blood lipid profile of submariners was examined.

Two cohorts of male RN submariners (SUB1: $n=32$; SUB2: $n=67$ ) had body mass measured immediately before departure and on return from a 3-month deployment. Skinfold measurements were also undertaken at the same time points on SUB1. Serum lipids and glucose were measured in blood samples taken after a 12-h fast, pre and post deployment. The study was approved by the Ministry of Defence Research Ethics Committee (Ref: 0903/228) and volunteers provided informed consent.

Mean body mass decreased in both groups (SUB1: $89.3 \pm 13$ vs. $85.6 \pm 10 \mathrm{~kg}$; SUB2: $85.4 \pm 15 \mathrm{vs.} 82.7 \pm 12 \mathrm{~kg}, \mathrm{P}<0.05$ ), and estimated body fat percentage decreased in SUB2 $(22 \pm 6$ vs. $21 \pm 5 \%, \mathrm{P}<0.05)$ pre vs. post deployment. All serum lipids in SUB1 and total cholesterol and HDL-cholesterol in SUB2 were significantly lower post deployment.

\begin{tabular}{|c|c|c|c|c|c|c|c|c|}
\hline & \multicolumn{4}{|l|}{ SUB1 } & \multicolumn{4}{|l|}{ SUB2 } \\
\hline & PRE & SD & POST & SD & PRE & SD & POST & SD \\
\hline Total cholesterol $\left(\mathrm{mmol} . \mathrm{l}^{-1}\right)$ & 4.9 & $1 \cdot 1$ & $4 \cdot 3^{\mathrm{a}}$ & $0 \cdot 9$ & $4 \cdot 5$ & $0 \cdot 8$ & $4 \cdot 4^{\mathrm{a}}$ & $0 \cdot 8$ \\
\hline HDL-cholesterol $\left(\mathrm{mmol}^{-1^{-1}}\right)$ & 1.47 & $0 \cdot 3$ & $1 \cdot 29^{\mathrm{a}}$ & $0 \cdot 32$ & $1 \cdot 34$ & $0 \cdot 3$ & $1 \cdot 30^{\mathrm{a}}$ & $0 \cdot 2$ \\
\hline LDL-cholesterol $\left(\mathrm{mmol.1} \mathrm{l}^{-1}\right)$ & $2 \cdot 78$ & $1 \cdot 0$ & $2 \cdot 54^{\mathrm{a}}$ & $0 \cdot 8$ & $2 \cdot 59$ & $0 \cdot 9$ & $2 \cdot 65$ & 0.7 \\
\hline Triglyceride $\left(\mathrm{mmol} .1^{-1}\right)$ & 1.42 & $0 \cdot 9$ & $0.97^{\mathrm{b}}$ & $0 \cdot 5$ & 1.23 & $0 \cdot 5$ & 0.94 & $0 \cdot 4$ \\
\hline Glucose $\left(\mathrm{mmol} . \mathrm{l}^{-1}\right)$ & $5 \cdot 5$ & $0 \cdot 9$ & $5 \cdot 2^{\mathrm{b}}$ & $1 \cdot 1$ & 4.9 & $0 \cdot 4$ & $4 \cdot 9$ & $0 \cdot 4$ \\
\hline
\end{tabular}

${ }^{\mathrm{a}} \mathrm{P}<0.05$ Paired samples t-test; ${ }^{\mathrm{b}} \mathrm{P}<0.05$ Wilcoxon Signed Rank Test

This study shows lower serum lipids following a submarine deployment in conjunction with a mean decrease in body mass, which was contrary to the expected hypothesis, given the constraints of the occupation. However, these results are in agreement with the limited existing literature on submariners. ${ }^{(2,3)}$ It is unclear whether changes in serum lipids are associated with individual changes in body mass and/or activity levels, therefore further analysis should investigate these separate effects to better understand any potential metabolic health risk after prolonged submersion.

1. O'Neill S. and O'Driscoll L. (2015) Obesity Reviews 16:1-12.

2. Gasier H.G. et al., (2016) Nutrients 8 (2):E85.

3. Gasier et al., (2014) Calcified Tissue International 95(3):229-39. 